Bull. Korean Math. Soc. 48 (2011), No. 6, pp. 1225-1233

http://dx.doi.org/10.4134/BKMS.2011.48.6.1225

\title{
CONNECTEDNESS AND COMPACTNESS \\ OF WEAK EFFICIENT SOLUTIONS FOR VECTOR EQUILIBRIUM PROBLEMS
}

\author{
Xian Jun Long and Jian Wen Peng
}

\begin{abstract}
In this paper, without assumption of monotonicity, we study the compactness and the connectedness of the weakly efficient solutions set to vector equilibrium problems by using scalarization method in locally convex spaces. Our results improve the corresponding results in [X. H. Gong, Connectedness of the solution sets and scalarization for vector equilibrium problems, J. Optim. Theory Appl. 133 (2007), 151-161].
\end{abstract}

\section{Introduction}

It is well-known that the vector equilibrium problem provides a unified mathematical model for a wide range of practical problems, which includes as special cases vector optimization problems, vector variational inequality problems, vector complementarity problems and vector saddle point problems. In recent years, a large number of researches have been devoted to the existence of solutions for various kinds of vector equilibrium problems (see, for example, $[1,3,6,7,10,13]$ and the references therein).

On the other hand, one of the most important problems of vector variational inequalities and vector equilibrium problems is to investigate the properties of the solutions set. Among many desirable properties of the solutions set, the connectedness is of considerable interest, due to it provides the possibility of continuously moving from one solution to any other solution. In [12], Lee et al. investigated the path-connectedness of the set of weakly efficient solutions and the set of efficient solutions for vector variational inequalities in finite-dimensional spaces. In [4], Cheng discussed the connectedness of the set

Received July 7, 2010.

2010 Mathematics Subject Classification. 49J40, 90C31.

Key words and phrases. vector equilibrium problem, weak efficient solution, scalarization, existence, connectedness.

This work was supported by the National Natural Science Foundation of China (11001287, 11171363, 10871217), the Natural Science Foundation Project of Chongqing (CSTC 2010BB9254, CSTC 2009BB8240 and CSTC 2009BB3372), the Education Committee Project Research Foundation of Chongqing (KJ100711). 
of weakly efficient solutions for weak vector variational inequalities in finitedimensional spaces by using scalarization method. In [7], Gong obtained the connectedness of the set of Henig efficient solutions and the set of weak efficient solutions to the vector-valued Hartman-Stampacchia variational inequality in normed spaces by using scalarization method. Recently, Gong [8] introduced the concepts of $f$-efficient solution, Henig efficient solution, globally efficient solution, weakly efficient solution, superefficient solution and cone-Benson efficient solution to vector equilibrium problems and gave some scalarization characterization for various proper efficient solutions. By using the scalarization results, he investigated the connectedness of the Henig efficient solutions set, globally efficient solutions set, weakly efficient solutions set, superefficient solutions set and cone-Benson efficient solutions set for vector equilibrium problems in locally convex spaces. Very recently, by virtue of a density result and scalarization technique, Gong and Yao [9] first discussed the connectedness of the set of efficient solutions for generalized systems in locally convex spaces.

In above mentioned works, the monotonicity plays an important role in deriving the connectedness of the sets of various proper efficient solutions to vector equilibrium problems and vector variational inequality problems. In this paper, without assumption of monotonicity, we obtain the compactness and the connectedness of the weakly efficient solutions set for vector equilibrium problems in locally convex spaces. Our results improve the corresponding ones of Gong [8].

\section{Preliminaries}

Throughout this paper, let $X$ be a real Hausdorff topological vector space and let $Y$ be a real locally convex Hausdorff topological vector space. Let $K$ be a nonempty closed convex subset of $X$. Let $C$ be a pointed closed convex cone $Y$. Let $Y^{*}$ be the topological dual space of $Y$, and $C^{*}=\left\{f \in Y^{*}: f(x) \geq\right.$ 0 for all $x \in C$ \} be the dual cone of $C$.

Denote the quasi-interior of $C^{*}$ by $C^{\sharp}$, i.e.,

$$
C^{\sharp}:=\left\{f \in Y^{*}: f(y)>0 \text { for all } y \in C \backslash\{0\}\right\} .
$$

Let $D$ be a nonempty subset of $Y$. The cone hull of $D$ is defined by

$$
\text { cone }(D)=\{t d: t \geq 0, d \in D\} .
$$

Denote the closure of $D$ by $\operatorname{cl}(D)$ and the interior of $D$ by $\operatorname{int} D$. A nonempty convex subset $B$ of $C$ is called a base of $C$ if $C=\operatorname{cone}(B)$ and $0 \notin \operatorname{cl}(B)$. It is easy to see that $C^{\sharp} \neq \emptyset$ if and only if $C$ has a base. Set

$$
C^{\triangle}=\left\{f \in C^{\sharp}: \text { there exists } t>0 \text { such that } f(b) \geq t \text { for all } b \in B\right\} .
$$

By the separation theorem of convex sets, we know that $C^{\triangle} \neq \emptyset$. Obviously, $C^{\triangle} \subset C^{\sharp}$. Let $B$ be a base of $C$. Then, $0 \notin \operatorname{cl}(B)$. By the separation theorem of convex sets, there exists $f \in Y^{*} \backslash\{0\}$ such that

$$
r=\inf \{f(b): b \in B\}>f(0)=0 .
$$


Set $V_{B}=\left\{y \in Y:|f(y)|<\frac{r}{2}\right\}$. It follows that $V_{B}$ is an open convex circled neighborhood of 0 in $Y$. It is clear that

$$
\inf \left\{f(y): y \in B+V_{B}\right\} \geq \frac{r}{2} \text {. }
$$

Note that for any convex neighborhood $U$ of 0 with $U \subset V_{B}, B+U$ is a convex set and $0 \notin \operatorname{cl}(B+U)$. Therefore, $C_{U}(B):=\operatorname{cone}(B+U)$ is a pointed convex cone and $C \backslash\{0\} \subset \operatorname{int} C_{U}(B)$.

Let $F: K \times K \rightarrow Y$ be a vector-valued mapping. Gong [7,8] considered the following vector equilibrium problem (in short, VEP): finding $x \in K$ such that

$$
F(x, y) \notin-A \text { for all } y \in K,
$$

where $A \cup\{0\}$ is a convex cone in $Y$.

We recall some definitions and lemmas which will be used later.

Definition $2.1([7])$. Let $\operatorname{int} C \neq \emptyset$. A vector $x \in K$ is called a weakly efficient solution to the (VEP) if

$$
F(x, y) \notin-\operatorname{int} C \text { for all } y \in K .
$$

Denote by $V_{W}(K, F)$ the set of all weakly efficient solutions to the (VEP).

Definition $2.2([7])$. Let $f \in C^{*} \backslash\{0\}$. A vector $x \in K$ is called a $f$-efficient solution to the (VEP) if

$$
f(F(x, y)) \geq 0 \text { for all } y \in K .
$$

Denote by $V_{f}(K, F)$ the set of all $f$-efficient solutions to the (VEP).

Definition 2.3 ([5]). A set-valued mapping $G: K \rightarrow 2^{X}$ is called a KKMmapping if, for any finite subset $\left\{x_{1}, x_{2}, \ldots, x_{n}\right\}$ of $K, \operatorname{co}\left\{x_{1}, x_{2}, \ldots, x_{n}\right\}$ is contained in $\bigcup_{i=1}^{n} G\left(x_{i}\right)$, where co $A$ denotes the convex hull of the set $A$.

Lemma 2.1 ([5]). Let $M$ be a nonempty subset of $X$ and $G: M \rightarrow 2^{X}$ be a KKM-mapping. If $G(x)$ is closed for any $x \in M$ and is compact for at least one $x \in M$, then $\bigcap_{y \in M} G(y) \neq \emptyset$.

Definition 2.4 ([14]). A vector-valued mapping $h: K \rightarrow Y$ is said to be $C$-convex on $K$ if, for any $x_{1}, x_{2} \in K$ and $\lambda \in[0,1]$, one has

$$
\lambda h\left(x_{1}\right)+(1-\lambda) h\left(x_{2}\right) \in h\left(\lambda x_{1}+(1-\lambda) x_{2}\right)+C .
$$

Remark 2.1. (i) It is easy to see that $h$ is $C$-convex on $K$ if and only if for any $x_{i} \in K$ and $\lambda_{i} \in[0,1](i=1,2, \ldots, n)$ with $\sum_{i=1}^{n} \lambda_{i}=1$, we have

$$
\sum_{i=1}^{n} \lambda_{i} h\left(x_{i}\right) \in h\left(\sum_{i=1}^{n} \lambda_{i} x_{i}\right)+C .
$$

(ii) If $h$ is $C$-convex on $K$, then $h(K)+C$ is a convex set.

(iii) If $-h$ is $C$-convex on $K$, then $h$ is said to be $C$-concave on $K$.

(iv) If $f \in C^{*} \backslash\{0\}$ and $h$ is $C$-convex on $K$, then $f \circ h: K \rightarrow R$ is convex.

(v) If $h_{1}, h_{2}: K \rightarrow Y$ are two $C$-convex vector-valued mappings on $K$, then $h_{1}+h_{2}$ is $C$-convex on $K$; see [14, Proposition 6.7]. 
Definition 2.5 ([14]). A vector-valued mapping $h: K \rightarrow Y$ is said to be $C$-lower ( $C$-upper) semicontinuous at $x_{0} \in K$ if, for any neighborhood $U$ of 0 , there exists a neighborhood $U\left(x_{0}\right)$ of $x_{0}$ such that

$$
\begin{gathered}
h(x) \in h\left(x_{0}\right)+U+C \text { for all } x \in U\left(x_{0}\right) \cap K \\
\left(h(x) \in h\left(x_{0}\right)+U-C \text { for all } x \in U\left(x_{0}\right) \cap K\right) .
\end{gathered}
$$

$h$ is said to be $C$-lower ( $C$-upper) semicontinuous on $K$ if it is $C$-lower $(C$ upper) semicontinuous at all $x_{0} \in K$.

Remark 2.2. If $f \in C^{*} \backslash\{0\}$ and $h$ is $C$-lower ( $C$-upper) semicontinuous on $K$, then $f \circ h: K \rightarrow R$ is lower (upper) semicontinuous on $K$; see [3].

Definition 2.6 ([2]). Let $S: K \rightarrow 2^{Y}$ be a set-valued mapping.

(i) $S$ is said to be upper semicontinuous at $x \in K$ if, for any open set $V$ containing $S(x)$, there exists an open set $U$ containing $x$ such that, for all $t \in U, S(t) \subset V$; $S$ is said to be upper semicontinuous on $K$ if it is upper semicontinuous at all $x \in K$.

(ii) $S$ is said to be closed if $\operatorname{Graph}(S)=\{(x, y): x \in K$ and $y \in S(x)\}$ is a closed set in $K \times Y$.

Lemma 2.2 ([2]). Let $S: K \rightarrow 2^{Y}$ be a set-valued mapping. If $S$ is closed and $Y$ is compact, then $S$ is upper semicontinuous.

Lemma $2.3([7])$. Suppose that int $C \neq \emptyset$ and that $F(x, K)+C$ is a convex set for each $x \in K$. Then

$$
V_{W}(K, F)=\bigcup_{f \in C^{*} \backslash\{0\}} V_{f}(K, F) .
$$

\section{Main results}

In this section, we shall discuss the compactness and the connectedness of the weakly efficient solutions set to the vector equilibrium problem by using the scalarization results. Let $\varphi: K \times K \rightarrow Y$ and $\psi: K \rightarrow Y$ be two vectorvalued mappings. In the sequel, unless specified otherwise, we assume that $F(x, y)=\varphi(x, y)+\psi(y)-\psi(x)$

First, we have the following existence results for the (VEP).

Theorem 3.1. Let $\operatorname{int} C \neq \emptyset$. Suppose that the following conditions are satisfied:

(i) for any $x \in K, \varphi(x, x) \in C$ and $\varphi(x, \cdot)$ is $C$-convex on $K$;

(ii) $\psi$ is $C$-lower semicontinuous and $C$-convex on $K$;

(iii) for any $y \in K, \varphi(\cdot, y)$ are $C$-upper semicontinuous and $C$-concave on $K$;

(iv) there exist a nonempty compact convex subset $E$ of $K$ and $y_{0} \in E$ such that any $x \in K \backslash E$ satisfies $\varphi\left(x, y_{0}\right)+\psi\left(y_{0}\right)-\psi(x) \in-\operatorname{int} C$.

Then, for any $f \in C^{*} \backslash\{0\}, V_{f}(K, F)$ is a nonempty compact convex subset of E. 
Proof. Let $f \in C^{*} \backslash\{0\}$. Define a set-valued mappings $A: K \rightarrow 2^{K}$ by

$$
A(y)=\{x \in K: f(\psi(y))+f(\varphi(x, y)) \geq f(\psi(x))\}, \forall y \in K .
$$

By assumption (i), we have $y \in A(y)$. Then, $A(y) \neq \emptyset$ for any $y \in K$. First, we prove that $A$ is a KKM-mapping. In fact, suppose by contradiction that there exist a finite subset $\left\{y_{1}, y_{2}, \ldots, y_{n}\right\}$ of $K$ and $\lambda_{i} \geq 0, i=1,2, \ldots, n$ with $\sum_{i=1}^{n} \lambda_{i}=1$ such that $x=\sum_{i=1}^{n} \lambda_{i} y_{i} \notin \bigcup_{i=1}^{n} A\left(y_{i}\right)$. Then, $x \notin A\left(y_{i}\right)$, $i=1,2, \ldots, n$. It follows that

$$
f\left(\psi\left(y_{i}\right)\right)+f\left(\varphi\left(x, y_{i}\right)\right)<f(\psi(x)), i=1,2, \ldots, n,
$$

which implies that

$$
\begin{aligned}
f\left(\sum_{i=1}^{n} \lambda_{i} \psi\left(y_{i}\right)\right)+f\left(\sum_{i=1}^{n} \lambda_{i} \varphi\left(x, y_{i}\right)\right) & =\sum_{i=1}^{n} \lambda_{i} f\left(\psi\left(y_{i}\right)\right)+\sum_{i=1}^{n} \lambda_{i} f\left(\varphi\left(x, y_{i}\right)\right) \\
& <f(\psi(x)) .
\end{aligned}
$$

Since $\psi$ and $\varphi(x, \cdot)$ are $C$-convex,

$$
\begin{aligned}
f\left(\sum_{i=1}^{n} \lambda_{i} \psi\left(y_{i}\right)\right)+f\left(\sum_{i=1}^{n} \lambda_{i} \varphi\left(x, y_{i}\right)\right) & \geq f\left(\psi\left(\sum_{i=1}^{n} \lambda_{i} y_{i}\right)\right)+f\left(\varphi\left(x, \sum_{i=1}^{n} \lambda_{i} y_{i}\right)\right) \\
& =f(\psi(x))+f(\varphi(x, x)) \\
& \geq f(\psi(x)),
\end{aligned}
$$

which contradicts (3.1). Thus, $A$ is a KKM-mapping.

Next, we prove that for any $y \in K, A(y)$ is closed. Indeed, for any fixed $y \in K$, let $\left\{x_{\alpha}\right\} \subset A(y)$ such that $x_{\alpha} \rightarrow x_{0}$. By the closedness of $K$, we have $x_{0} \in K$. Since $\left\{x_{\alpha}\right\} \subset A(y)$, one has

$$
f(\psi(y))+f\left(\varphi\left(x_{\alpha}, y\right)\right) \geq f\left(\psi\left(x_{\alpha}\right)\right) .
$$

Since $\varphi(\cdot, y)$ is $C$-upper semicontinuous and $\psi$ is $C$-lower semicontinuous,

$$
\begin{aligned}
f(\psi(y))+f\left(\varphi\left(x_{0}, y\right)\right) & \geq f(\psi(y))+\limsup f\left(\varphi\left(x_{\alpha}, y\right)\right) \\
& \geq \limsup f\left(\psi\left(x_{\alpha}\right)\right) \\
& \geq \liminf f\left(\psi\left(x_{\alpha}\right)\right) \\
& \geq f\left(\psi\left(x_{0}\right)\right),
\end{aligned}
$$

which implies that $x_{0} \in A(y)$. Thus, for any $y \in K, A(y)$ is closed. By the assumption, $A\left(y_{0}\right)$ is closed and $A\left(y_{0}\right) \subset E$. Since $E$ is compact, $A\left(y_{0}\right)$ is compact. By Lemma 2.1, we obtain $\bigcap_{y \in K} A(y) \neq \emptyset$. Therefore, there exists $x \in \bigcap_{y \in K} A(y)$. Noting that $V_{f}(K, F)=\bigcap_{y \in K} A(y)$. It follows that $x \in V_{f}(K, F)$, i.e., $V_{f}(K, F) \neq \emptyset$. It is easy to see that $V_{f}(K, F) \subset E$ is a compact set.

Finally, we show that $V_{f}(K, F)$ is a convex set. Since $V_{f}(K, F)=\bigcap_{y \in K} A(y)$, we need only to prove that for any $y \in K, A(y)$ is convex. In fact, for any fixed 
$y \in K$, let $x_{1}, x_{2} \in A(y)$ and $\lambda \in[0,1]$. Then, $\lambda x_{1}+(1-\lambda) x_{2} \in K$ and

$$
\begin{aligned}
& f(\psi(y))+f\left(\varphi\left(x_{1}, y\right)\right) \geq f\left(\psi\left(x_{1}\right)\right), \\
& f(\psi(y))+f\left(\varphi\left(x_{2}, y\right)\right) \geq f\left(\psi\left(x_{2}\right)\right) .
\end{aligned}
$$

Multiplying both side of (3.2) by $\lambda$ and of (3.3) by $1-\lambda$, and adding them, we get

$$
f(\psi(y))+\lambda f\left(\varphi\left(x_{1}, y\right)\right)+(1-\lambda) f\left(\varphi\left(x_{2}, y\right)\right) \geq \lambda f\left(\psi\left(x_{1}\right)\right)+(1-\lambda) f\left(\psi\left(x_{2}\right)\right) .
$$

This fact together with the $C$-convexity of $\psi$ and the $C$-concavity of $\varphi$ with respect to the first argument yields

$$
f(\psi(y))+f\left(\varphi\left(\lambda x_{1}+(1-\lambda) x_{2}, y\right)\right) \geq f\left(\psi\left(\lambda x_{1}+(1-\lambda) x_{2}\right)\right) .
$$

It follows that $\lambda x_{1}+(1-\lambda) x_{2} \in A(y)$. Therefore, for any $y \in K, A(y)$ is convex. And so $V_{f}(K, F)$ is convex. This completes the proof.

Now we establish the connectedness of the weakly efficient solution sets to the (VEP).

Theorem 3.2. Let $\operatorname{int} C \neq \emptyset, 0 \in K, \psi(0)=0$ and $\varphi(0,0)=0$. Let $\psi(K)$ and $D=\{\varphi(x, y): x, y \in K\}$ be two bounded subsets of $Y$. Assume that the conditions (i)-(iv) of Theorem 3.1 hold. Then, $V_{W}(K, F)$ is a connected set.

Proof. Define the set-valued mapping $H: C^{*} \backslash\{0\} \rightarrow 2^{E}$ by

$$
H(f)=V_{f}(K, F), f \in C^{*} \backslash\{0\} .
$$

By Theorem 3.1, for any $f \in C^{*} \backslash\{0\}, V_{f}(K, F) \subset E$ is a nonempty convex set. It follows that for any $f \in C^{*} \backslash\{0\}, H(f)$ is a connected set. It is clear that $C^{*} \backslash\{0\}$ is convex, so it is a connected set.

Now we show that $H(f)$ is upper semicontinuous on $C^{*} \backslash\{0\}$. Since $E$ is compact, by Lemma 2.2 , we need only to prove that $H$ is closed. Let $\left\{\left(f_{\alpha}, x_{\alpha}\right)\right.$ : $\alpha \in I\}$ be a net such that

$$
\left\{\left(f_{\alpha}, x_{\alpha}\right): \alpha \in I\right\} \subset \operatorname{Graph}(H)=\left\{(f, x) \in\left(C^{*} \backslash\{0\}\right) \times E: x \in H(f)\right\}
$$

and

$$
\left(f_{\alpha}, x_{\alpha}\right) \rightarrow\left(f, x_{0}\right) \in\left(C^{*} \backslash\{0\}\right) \times E
$$

where $f_{\alpha} \rightarrow f$ means that $\left\{f_{\alpha}\right\}$ converges to $f$ with respect to the strong topology $\beta\left(Y^{*}, Y\right)$ in $Y^{*}$. Since $x_{\alpha} \in H\left(f_{\alpha}\right), \alpha \in I$, one has

$$
f_{\alpha}(\psi(y))+f_{\alpha}\left(\varphi\left(x_{\alpha}, y\right)\right) \geq f_{\alpha}\left(\psi\left(x_{\alpha}\right)\right), \forall y \in K
$$

By assumption, $\psi(K)$ and $D=\{\varphi(x, y): x, y \in K\}$ are bounded subsets of $Y$. Define

$$
P_{\psi(K)+D}\left(y^{*}\right):=\sup \left\{\left|y^{*}(u)\right|: u \in \psi(K)+D\right\}, y^{*} \in Y^{*} .
$$

It is easy to see that $P_{\psi(K)+D}$ is a seminorm of $Y^{*}$. For arbitrary $\varepsilon>0$,

$$
U=\left\{y^{*} \in Y^{*}: P_{\psi(K)+D}\left(y^{*}\right)<\varepsilon\right\}
$$


CONNECTEDNESS AND COMPACTNESS OF WEAK EFFICIENT SOLUTIONS 1231

is a neighborhood of zero with respect to $\beta\left(Y^{*}, Y\right)$. Since $f_{\alpha}-f \rightarrow 0$, there exists $\alpha_{0} \in I$ such that $f_{\alpha}-f \in U, \forall \alpha \geq \alpha_{0}$. It follows that

$P_{\psi(K)+D}\left(f_{\alpha}-f\right)=\sup \left\{\left|\left(f_{\alpha}-f\right)(u)\right|: u \in \psi(K)+D\right\}<\varepsilon$, whenever $\alpha \geq \alpha_{0}$.

Therefore, for any $y \in K$,

$$
\begin{gathered}
\left|\left(f_{\alpha}-f\right)\left(\varphi\left(x_{\alpha}, y\right)\right)\right|=\left|f_{\alpha}\left(\varphi\left(x_{\alpha}, y\right)\right)-f\left(\varphi\left(x_{\alpha}, y\right)\right)\right|<\varepsilon, \\
\left|\left(f_{\alpha}-f\right)\left(\psi\left(x_{\alpha}\right)\right)\right|=\left|f_{\alpha}\left(\psi\left(x_{\alpha}\right)\right)-f\left(\psi\left(x_{\alpha}\right)\right)\right|<\varepsilon,
\end{gathered}
$$

and

$$
\left|f_{\alpha}(\psi(y))-f(\psi(y))\right|<\varepsilon \text { for all } \alpha \geq \alpha_{0},
$$

because $0 \in K, \psi(0)=0$ and $\varphi(0,0)=0$. It follows that for any $y \in K$,

$$
\begin{gathered}
\lim \left[f_{\alpha}\left(\varphi\left(x_{\alpha}, y\right)\right)-f\left(\varphi\left(x_{\alpha}, y\right)\right)\right]=0, \\
\lim \left[f_{\alpha}\left(\psi\left(x_{\alpha}\right)\right)-f\left(\psi\left(x_{\alpha}\right)\right)\right]=0,
\end{gathered}
$$

and

$$
\lim \left[f_{\alpha}(\psi(y))-f(\psi(y))\right]=0 .
$$

From (3.4), we have

$$
\begin{aligned}
& f_{\alpha}(\psi(y))+\left[f_{\alpha}\left(\varphi\left(x_{\alpha}, y\right)\right)-f\left(\varphi\left(x_{\alpha}, y\right)\right)\right]-\left[f_{\alpha}\left(\psi\left(x_{\alpha}\right)\right)-f\left(\psi\left(x_{\alpha}\right)\right)\right] \\
\geq & f\left(\psi\left(x_{\alpha}\right)\right)-f\left(\varphi\left(x_{\alpha}, y\right)\right),
\end{aligned}
$$

which implies that

$$
\begin{aligned}
& \lim f_{\alpha}(\psi(y))+\lim \left[f_{\alpha}\left(\varphi\left(x_{\alpha}, y\right)\right)-f\left(\varphi\left(x_{\alpha}, y\right)\right)\right] \\
& -\lim \left[f_{\alpha}\left(\psi\left(x_{\alpha}\right)\right)-f\left(\psi\left(x_{\alpha}\right)\right)\right] \\
\geq & \liminf \left[f\left(\psi\left(x_{\alpha}\right)\right)-f\left(\varphi\left(x_{\alpha}, y\right)\right)\right] .
\end{aligned}
$$

Noting that $\psi$ is $C$-lower semicontinuous and $\varphi(\cdot, y)$ is $C$-upper semicontinuous. This fact together with (3.5), (3.6), (3.7) and (3.8) yields

$$
\begin{aligned}
f(\psi(y))= & \lim f_{\alpha}(\psi(y))+\lim \left[f_{\alpha}\left(\varphi\left(x_{\alpha}, y\right)\right)-f\left(\varphi\left(x_{\alpha}, y\right)\right)\right] \\
& -\lim \left[f_{\alpha}\left(\psi\left(x_{\alpha}\right)\right)-f\left(\psi\left(x_{\alpha}\right)\right)\right] \\
\geq & \liminf \left[f\left(\psi\left(x_{\alpha}\right)\right)-f\left(\varphi\left(x_{\alpha}, y\right)\right)\right] \\
\geq & \liminf f\left(\psi\left(x_{\alpha}\right)\right)-\lim \sup f\left(\varphi\left(x_{\alpha}, y\right)\right) \\
\geq & f\left(\psi\left(x_{0}\right)\right)-f\left(\varphi\left(x_{0}, y\right)\right) .
\end{aligned}
$$

It follows that $x_{0} \in V_{f}(K, F)=H(f)$. Therefore, $H$ is a closed mapping, and so $H$ is upper semicontinuous on $C^{*} \backslash\{0\}$. From Theorem 3.1 in [11],

$$
\bigcup_{f \in C^{*} \backslash\{0\}} V_{f}(K, F)
$$


is a connected set. Furthermore, by the assumptions and Remark 2.1(v), we have, for any $x \in K, \psi(\cdot)+\varphi(x, \cdot)$ is $C$-convex on $K$. Hence, $\psi(K)+\varphi(x, K)+C$ is a convex set. By Lemma 2.3(i),

$$
V_{W}(K, F)=\bigcup_{f \in C^{*} \backslash\{0\}} V_{f}(K, F)
$$

is a connected set. This completes the proof.

Remark 3.1. If the condition (iv) in Theorem 3.1 (Theorem 3.2) is replaced by the condition that $K$ is a nonempty compact convex subset of $X$, then the conclusion still holds.

Remark 3.2. The following example illustrates that Theorems 3.1 and 3.2, respectively, improve Theorems 3.1 and 4.5 in [8] by removing the monotonicity of $\varphi$ and the $C$-lower semicontinuity of $\varphi$ with respect to the second argument.

Example 3.1. Let $X=\mathbb{R}, Y=\mathbb{R}^{2}, K=[0,1]$ and $C=\mathbb{R}_{+}^{2}$. Let

$$
\varphi(x, y)=(2 x-y, 2 x-y), \quad \psi(x)=(0,0) \text { for all } x, y \in K .
$$

It is easy to see that the assumptions of Theorem 3.1 are satisfied. By Theorems 3.1 and $3.2, V_{W}(K, F)=\left[\frac{1}{2}, 1\right]$ is a compact and connected set. However, neither Theorem 3.1 nor Theorem 4.5 in [8] is not applicable because $\varphi$ is not $C$-monotone (i.e., for any $x, y \in K, \varphi(x, y)+\varphi(y, x) \in-C$ ) on $K$. Indeed, let $x=1, y=1$, we have

$$
\begin{aligned}
\varphi(x, y)+\varphi(y, x) & =(2 x-y, 2 x-y)+(2 y-x, 2 y-x) \\
& =(x+y, x+y)=(2,2) \\
& \notin-C,
\end{aligned}
$$

which means $\varphi$ is not $C$-monotone on $K$.

\section{References}

[1] Q. H. Ansari and J. C. Yao, An existence result for the generalized vector equilibrium, Appl. Math. Lett. 12 (1999), no. 8, 53-56.

[2] J. P. Aubin and I. Ekeland, Applied Nonlinear Analysis, John Wiley, New York, 1984.

[3] M. Bianchi, N. Hadjisavvas, and S. Schaibles, Vector equilibrium problems with generalized monotone bifunctions, J. Optim. Theory Appl.92 (1997), no. 3, 527-542.

[4] Y. H. Cheng, On the connectedness of the solution set for the weak vector variational inequality, J. Math. Anal. Appl. 260 (2001), no. 1, 1-5.

[5] K. Fan, A generalization of Tychonoffs fixed point theorem, Math. Ann. 142 (1961), 305-310.

[6] F. Giannessi, (ed.), Vector Variational Inequilities and Vector Equilibria: Mathematical Theories, Kluwer, Dordrechet, 2000.

[7] X. H. Gong, Efficiency and Henig efficiency for vector equilibrium problems, J. Optim. Theory Appl. 108 (2001), no. 1, 139-154.

[8] _ Connectedness of the solution sets and scalarization for vector equilibrium problems, J. Optim. Theory Appl. 133 (2007), no. 2, 151-161.

[9] X. H. Gong and J. C. Yao, Connectedness of the set of efficient solutions for generalized systems, J. Optim. Theory Appl. 138 (2008), no. 2, 189-196. 
CONNECTEDNESS AND COMPACTNESS OF WEAK EFFICIENT SOLUTIONS 1233

[10] N. Hadjisavvas and S. Schaibles, From scalar to vector equilibrium problems in the quasimonotone case, J. Optim. Theory Appl. 96 (1998), no. 2, 297-309.

[11] Y. D. Hu, The efficiency Theory of Multiobjective Programming, Shanghai: Shanghai Science and Technology Press, 1994

[12] G. M. Lee, D. S. Kim, B. S. Lee, and N. D. Yun, Vector variational inequality as a tool for studying vector optimization problems, Nonlinear Anal. 34 (1998), no. 5, 745-765.

[13] X. J. Long, N. J. Huang, and K. L. Teo, Existence and stability of solutions for generalized strong vector quasi-equilibrium problems, Math. Comput. Modelling 47 (2008), no. 3-4, 445-451.

[14] D. T. Luc, Theory of Vector Optimization, Lecture Notes in Economics and Mathematics Systems, Vol. 319, Springer-Verlag, New York, 1989

XIAN JUN LONG

College of Mathematics and Statistics

Chongling Technology and Business University

Chongqing 400067, P. R. China

E-mail address: xianjunlong@hotmail.com

JIAN WEN PENG

Department of Mathematics

Chongqing Normal University

Chongqing 400047, P. R. China

E-mail address: jwpeng6@yahoo.com.cn 